\title{
Спинодальный распад в InSb/AlAs-гетероструктурах
}

\author{
(C) Д.С. Абрамкин ${ }^{1,2}$, А.К. Бакаров ${ }^{1}$, А.К. Гутаковский ${ }^{1,2}$, Т.С. Шамирзаев ${ }^{1,2,3}$ \\ ${ }^{1} 1$ Институт фризики полупроводников им. А.В. Ржанова Сибирского отделения Российской академии наук, \\ 630090 Новосибирск, Россия \\ ${ }^{2}$ Новосибирский государственный университет, \\ 630090 Новосибирск, Россия \\ ${ }^{3}$ Уральский фредеральный университет, \\ 620002 Екатеринбург, Россия \\ E-mail: demid@isp.nsc.ru
}

(Получена 25 апреля 2018 г. Принята к печати 7 мая 2018 г.)

Изучено атомное строение и энергетический спектр гетероструктур, формирующихся в системе бинарных соединений InSb/AlAs. Твердый раствор $\operatorname{In}_{x} \mathrm{Al}_{1-x} \mathrm{Sb}_{y} \mathrm{As}_{1-y}$, из которого формируются квантовые ямы в структураx InSb/AlAs, распадается на две фазы разного состава. Характерные размеры областей, содержащих отдельные фазы твердого раствора в плоскости структуры, составляют 5-7нм. Спинодальный распад твердого раствора приводит к формированию в квантовых ямах изученных гетероструктур сосуществующих областей с энергетическим спектром первого и второго рода и непрямой в пространстве квазиимпульсов запрещенной зоной.

DOI: $10.21883 /$ FTP.2018.11.46583.05

\section{1. Введение}

Полупроводниковые низкоразмерные гетероструктуры можно разделить по типу энергетического строения на структуры первого рода, в которых носители заряда различных знаков локализованы в одной области пространства, и на структуры второго рода, в которых носители заряда пространственно разделены. Для каждого типа структур в пространстве квазиимпульсов также могут реализоваться два варианта: (i) прямозонные структуры, когда электрон и дырка расположены в одной и той же точке зоны Бриллюэна, и (ii) непрямозонные структуры, образующиеся, когда носители заряда имеют разное значение квазиимпульса [1]. Разделение электронов и дырок в пространстве квазиимпульсов приводит к длительному времени жизни экситона [2], вплоть до 100 мкс, что оказывается сравнимым со временем спиновой релаксации локализованного экситона [3]. Это делает непрямозонные гетероструктуры перспективными объектами для исследования спиновой динамики долгоживущих локализованных экситонов $[4,5]$.

Как показывают теоретические расчеты [6,7], низкоразмерные структуры, формирующиеся в системе бинарных соединений InSb/AlAs, могут принадлежать малоисследованному классу непрямозонных систем первого рода. В наших недавних работах было показано, что в процессе эпитаксии таких гетероструктур осаждение InSb на поверхность AlAs сопровождается перемешиванием материалов, приводящим к формированию четверного твердого раствора $\operatorname{In}_{x} \mathrm{Al}_{1-x} \mathrm{Sb}_{y} \mathrm{As}_{1-y}$ [8]. Поскольку бинарные соединения InSb и AlAs сильно рассогласованны по параметру решетки (12\% [9]), при формировании твердых растворов на их основе можно ожидать спинодального распада - образования пространственно разделенных фаз с различным составом твердого раствора $[10,11]$. Однако детальные исследования строения гетероструктур InSb/AlAs с квантовыми ямами (КЯ) и его влияния на их энергетический спектр до сих пор отсутствуют.

Работа посвящена исследованию кристаллической структуры и энергетического спектра гетероструктур, формирующихся при эпитаксиальном росте одного монослоя (MC) InSb в матрице AlAs. Показано, что твердый раствор $\operatorname{In}_{x} \mathrm{Al}_{1-x} \mathrm{Sb}_{y} \mathrm{As}_{1-y}$, образующийся из-за перемешивания материалов в процессе эпитаксиального роста, распадается на пространственно разделенные фазы различного состава. Пространственная модуляция состава твердого раствора приводит к формированию в КЯ сосуществующих областей с непрямозонным энергетическим спектром первого и второго рода.

\section{2. Методика эксперимента}

Гетероструктуры InSb/AlAs с КЯ выращивались методом молекулярно-лучевой эпитаксии на подложках GaAs ориентации (001). На буферном слое GaAs выращивался слой AlAs толщиной 50 нм при температуре подложки $\left(T_{s}\right) 620^{\circ} \mathrm{C}$ и скорости осаждения материала 1 монослой в секунду (MC/c). Затем рост прекращался, а температура подложки снижалась до $500^{\circ} \mathrm{C}$. Во время снижения температуры структуры выдерживались в потоке $\mathrm{As}_{4}$. Дифракция быстрых электронов на отражение показывает, что поверхность AlAs после снижения температуры характеризовалась реконструкцией с $(4 \times 4)$. Осаждение слоя $\mathrm{InSb}$ проводилось со скоростью $0.1 \mathrm{MC} / \mathrm{c}$ в количестве, эквивалентном 1 MC. После формирования слой InSb заращивался слоем AlAs толщиной 50 нм без изменения температуры подложки. Верхний слой AlAs защищался от окисления слоем GaAs толщиной 20 нм.

Кристаллическое строение гетероструктур было исследовано методом просвечивающей электронной мик- 
роскопии (ПЭМ). Для получения ПЭМ изображений в режиме с $Z$-контрастом использовался просвечивающий электронный микроскоп JEM-4000ЕX (400 кэВ). Химическое картирование проведено с помощью рентгеновского энерго-дисперсионного анализа с использованием просвечивающего электронного микроскопа Titan 80-300 (FEI). Стационарная фотолюминесценция $(Ф Л)$ возбуждалась излучением $\mathrm{GaN}$ лазерного диода с энергией кванта 3.06 эВ и плотностью мощности $\left(P_{\text {ex }}\right)$, варьируемой в диапазоне 20 мкВт/см ${ }^{2}-2 \mathrm{Bт} / \mathrm{cm}^{2}$. Спектры ФЛ анализировались с помощью спектрографа Acton Advanced SP2500A и измерялись ПЗС-камерой с азотным охлаждением. Измерения проводились при температуре $5 \mathrm{~K}$. Время-разрешенная ФЛ возбуждалась полупроводниковым лазером с энергией кванта 3.49 эВ,

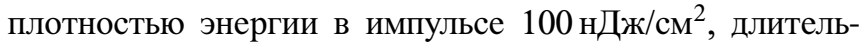
ностью импульсов 5 нс и частотой повторения 1 кГц.

\section{3. Экспериментальные результаты}

\section{1. Просвечивающая электронная микроскопия}

Планарное ПЭМ изображение InSb/AlAs-гетероструктуры, полученное в режиме с $Z$-контрастом, представленное на рис. 1, демонстрирует неоднородность в плоскости структуры. Отчетливо видны области характерного размера 5-7 нм с различным составом твердого раствора. На ПЭМ изображении поперечного среза гетероструктуры, показанном на вставке к рисунку, хорошо видно, что в структуре формируется КЯ толщиной 3 нм,

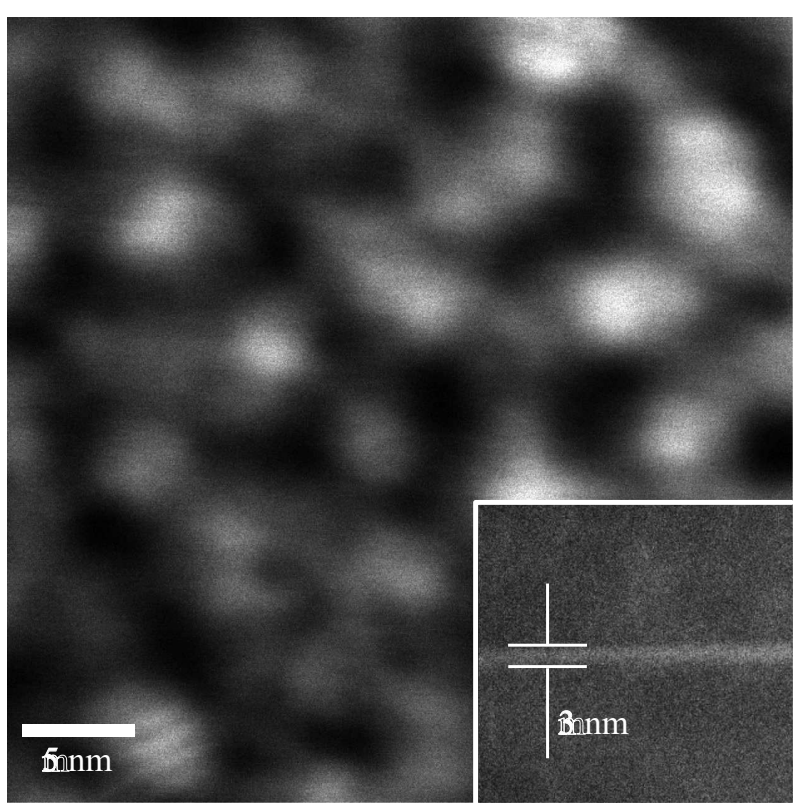

Рис. 1. Планарное ПЭМ изображение InSb/AlAs-гетероструктуры, полученное в режиме с Z-контрастом. На вставке представлено ПЭМ изображение поперечного среза гетероструктуры.

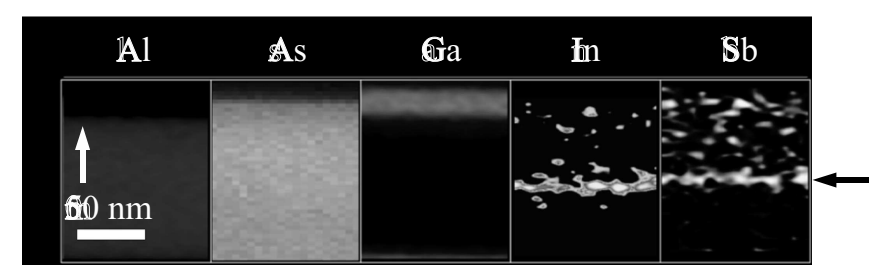

Рис. 2. ПЭМ изображения поперечного среза InSb/AlAs-гетероструктуры, полученные в режиме химического картирования. Направление роста гетероструктуры и положение КЯ обозначены соответственно вертикальной и горизонтальной стрелками.

что заметно превосходит толщину, соответствующую номинальному количеству осажденного InSb (1 MC). Уширение КЯ обусловлено перемешиванием материалов при осаждении InSb и(или) заращивании гетероструктуры AlAs и формировании КЯ из четверного твердого раствора $\operatorname{In}_{x} \mathrm{Al}_{1-x} \mathrm{Sb}_{y} \mathrm{As}_{1-y}$, что согласуется с результатами наших предыдущих работ $[8,12]$.

Поперечные срезы гетероструктуры InSb/AlAs, полученные в просвечивающем электронном микроскопе в режиме химического картирования, представленные на рис. 2, подтверждают формирование уширенной КЯ, состоящей из твердого раствора. Как видно из рисунка, содержание атомов In и Sb в верхнем слое AlAs заметно превосходит их содержание в нижнем слое AlAs, что указывает на сегрегацию In и $\mathrm{Sb}$ в ходе заращивания слоя $\mathrm{InSb}$ арсенидом алюминия.

\section{2. Фотолюминесценция}

Низкотемпературный $(5 \mathrm{~K})$ спектр стационарной ФЛ $\mathrm{InSb} / \mathrm{AlAs-гетероструктуры,} \mathrm{измеренный} \mathrm{при} \mathrm{плотности}$ мощности $P_{\text {ex }}=2 \mathrm{MBT} / \mathrm{cm}^{2}$, показан на верхней вставке к рис. 3. Спектр ФЛ состоит из двух полос, обозначенных как I и II, форма которых описывается функциями Гаусса, с максимумами 2.005 и 1.835 эВ и шириной на половинной интенсивности 46 и 90 мэВ соответственно. Сегрегация индия и сурьмы при заращивании тонкого слоя InSb арсенидом алюминия приводит к формированию квантовых ям с диффузно размытой гетерограницей [12]. Размытие гетерограницы сопровождается формированием в таких квантовых ямах хвостов плотности электронных состояний, связанных с флуктуациями ширины и состава КЯ. Большая ширина полос в спектрах ФЛ изучаемых КЯ обусловлена связанным с этими флуктуациями неоднородным уширением энергических уровней размерного квантования электронов и дырок.

Приведенные на нижней вставке к рис. 3 зависимости интегральных интенсивностей $(I)$ обеих полос от плотности мощности сохраняют линейный характер вПлоть до $P_{\text {ex }}=200 \mathrm{мBт} / \mathrm{cm}^{2}$, отмеченной на рисунке вертикальной стрелкой. При дальнейшем увеличении плотности мощности зависимости становятся сублинейными $I \sim P_{\text {ex }}^{0.7}$. Повышение плотности мощности сопро- 


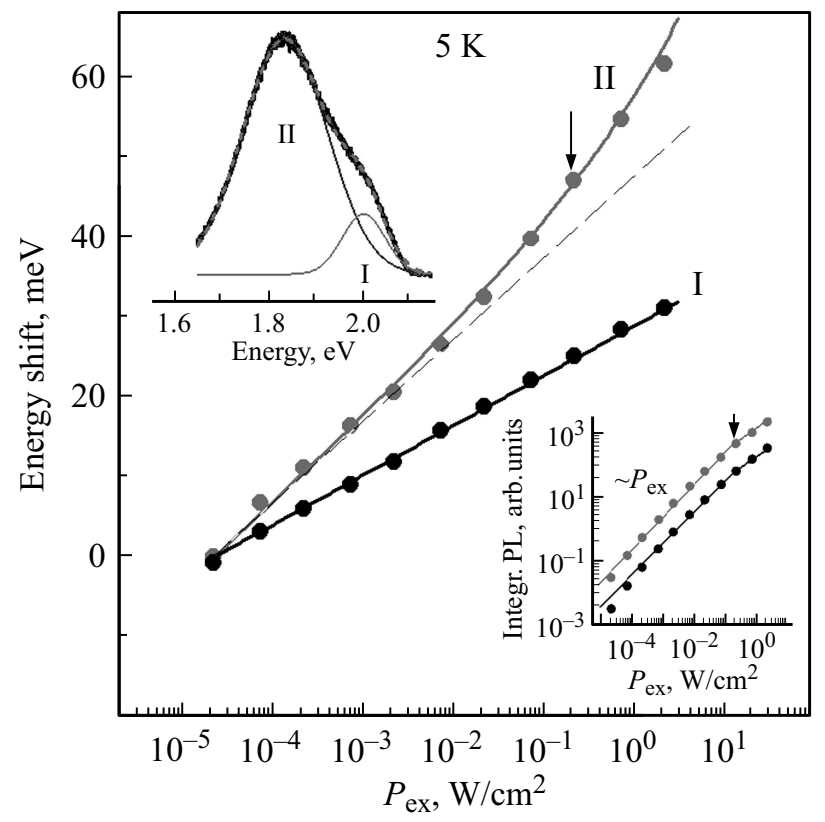

Рис. 3. Спектральное смещение положения максимума высоко- I и низкоэнергетической II полос стационарной ФЛ InSb/AlAs-гетероструктуры в зависимости от плотности возбуждения. Сплошные линии соответствуют аппроксимации экспериментальных данных выражением $U \ln \left(P_{\mathrm{ex}}\right)+b P_{\mathrm{ex}}^{1 / 3}$. На верхней вставке представлен спектр стационарной ФЛ InSb/AlAs-гетероструктуры, измеренный при температуре $5 \mathrm{~K}$ и $P_{\text {ex }}=2 \mathrm{мBт} /$ см $^{2}$. Аппроксимация спектра ФЛ двумя функциями Гаусса (сплошные серые линии), соответствующими полосам I и II, показана пунктирной серой линией. На нижней вставке изображены зависимости интегральной интенсивности ФЛ от плотности мощности возбуждения для обеих полос ФЛ. Вертикальными стрелками на рисунке и вставке отмечена плотность мощности $P_{\mathrm{ex}}=200 \mathrm{MBT} / \mathrm{cm}^{2}$, по достижении которой зависимость $I(P)$ становится сублинейной.

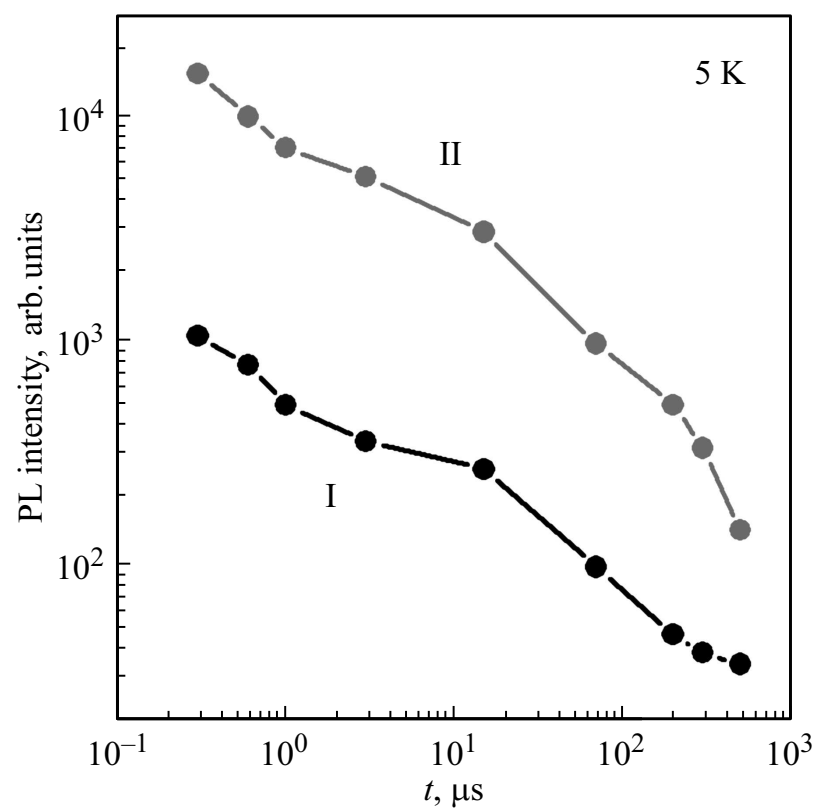

Рис. 4. Зависимости интегральной интенсивности полос ФЛ I и II от времени после окончания импульса возбуждения. Сплошные линии приведены для удобства восприятия. вождается также сдвигом положений максимумов полос ФЛ (показанных на рис. 3 как зависимости I и II) в высокоэнергетическую область спектра.

Зависимости интегральной интенсивности полос I и II, в спектре ФЛ, от времени после окончания лазерного импульса приведены на рис. 4. Затухание ФЛ обеих полос в течение сотен микросекунд после импульса возбуждения указывает на то, что в этих оптических переходах участвуют носители заряда, разделенные в пространстве квазиимпульсов [2].

\section{4. Обсуждение результатов}

Образование в плоскости структуры двух фаз различного состава вместо однородного четверного твердого раствора $\operatorname{In}_{x} \mathrm{Al}_{1-x} \mathrm{Sb}_{y} \mathrm{As}_{1-y}$, из которого формируется КЯ, указывает на его спинодальный распад. Поскольку упругие деформации, возникающие в ходе формирования КЯ и сегрегации материалов при ее заращивании, неоднородны, распределение фаз в плоскости структуры не является строго периодическим.

Наблюдаемые в спектрах ФЛ InSb/AlAs-гетероструктуры полосы мы связываем с рекомбинацией носителей заряда, локализованных в участках КЯ с различным составом твердого раствора. В нашей недавней работе [13] было показано, что энергетический спектр KT, формирующихся в гетеросистеме InAlSbAs/AlAs, в зависимости от состава твердого раствора может иметь энергетический спектр как первого, так и второго рода.

В отличие от квантовых точек, формирующихся по механизму Странского-Крастанова с резкими гетерограницами, в InAlSbAs/AlAs КЯ границы диффузно размыты, что приводит к неоднородному уширению их энергетического спектра. Для определения рода энергетического спектра в структурах с неоднородно уширенным спектром электронных состояний мы использовали развитую ранее нами в работах $[12,14]$ методику, основанную на измерении зависимостей интегральной интенсивности и положения максимума полос ФЛ от плотности мощности возбуждения. Смещение максимумов полос I и II описывалось выражением $U \ln \left(P_{\text {ex }}\right)+b P_{\text {ex }}^{1 / 3}$ в диапазоне линейной зависимости $I \sim P_{\text {ex. }}$ В этом выражении смещение $\sim P_{\mathrm{ex}}^{1 / 3}$ связано с эффектом искривления энергетических зон и вызванного им смещения уровней размерного квантования в системах с энергетическим строением второго рода, а смещение, описываемое логарифмическим членом, обусловлено заполнением хвостов плотности электронных состояний и не зависит от рода энергетического спектра гетероструктуры [12]. Результаты подгонки экспериментальных зависимостей этим выражением приведены на рис. 3 сплошными линиями, а значения параметров $U$ и $b$, наилучшим образом описывающие экспериментальные данные, представлены в таблице.

Видно, что смещение полосы I следует простому логарифмическому закону $\sim U \ln \left(P_{\mathrm{ex}}\right)$, характерному для 
Значения параметров $U$ и $b$, наилучшим образом описывающих экспериментальные зависимости спектрального смещения положений максимумов полос ФЛ при повышении плотности возбуждения

\begin{tabular}{c|c|c}
\hline Полоса ФЛ & $U$, мэВ & $b, \mathrm{мэB} /\left(\mathrm{BT} / \mathrm{cm}^{2}\right)^{1 / 3}$ \\
\hline I & $2.8 \pm 0.1$ & 0 \\
II & $4.4 \pm 0.1$ & $0.01 \pm 0.001$
\end{tabular}

гетероструктур первого рода с неоднородно уширенным спектром [12]. В то время как дополнительный вклад $b P_{\mathrm{ex}}^{1 / 3}$ в синее смещение максимума полосы II характерен для гетероструктур второго рода. Таким образом, спинодальный распад твердого раствора в InSb/AlAs-гетероструктуре с КЯ приводит к появлению двух областей с непрямозонным энергетическим спектром: (1) первого рода, ответственной за появление полосы ФЛ I и (2) второго рода, с которой связано появления полосы ФЛ II.

Для того чтобы оценить составы твердого раствора в областях с различным энергетическим спектром, были проведены модельные расчеты. Гетероструктура моделировалась как $\mathrm{In}_{x} \mathrm{Al}_{1-x} \mathrm{Sb}_{y} \mathrm{As}_{1-y}$ КЯ с расположенными в шахматном порядке областями, отличающимися составом твердого раствора. Распределение состава твердого раствора рассчитывалось в два этапа: сначала учитывался распад КЯ на области с различным составом, a затем принималась во внимание сегрегация In и $\mathrm{Sb}$. На первом этапе предполагалось, что атомы In и $\mathrm{Sb}$, общее количество которых соответствует $1 \mathrm{MC}$ $\mathrm{InSb}$, перераспределяются по участкам КЯ в одинаковых пропорциях, т.е. всюду $x=y$. Значения $x_{\max }=y_{\max }$, соответствуют участкам КЯ с высоким содержанием In и $\mathrm{Sb}$, a $x_{\min }=y_{\min }-\mathrm{c}$ низким. Состав твердого раствора изменялся в плоскости КЯ с периодом 5 нм. Сегрегационный профиль рассчитывался в соответствии с моделью, предложенной в работе Мураки [15]. Коэффициенты сегрегации для In и $\mathrm{Sb}$ в AlAs были взяты 0.78 [16] и 0.9 соответственно. Все постоянные решетки твердого раствора в обеих областях КЯ были согласованы с AlAs-матрицей в плоскости структуры. Поперечное сечение модельной структуры в области КЯ схематически представлено на рис. 5. Градация серого схематично отражает состав $\operatorname{In}_{x} \mathrm{Al}_{1-x} \mathrm{Sb}_{y} \mathrm{As}_{1-y}$, области с высоким содержанием In и $\mathrm{Sb}$ отмечены на рисунке как B, с низким - как A.

Параметры твердого раствора $\operatorname{In}_{x} \mathrm{Al}_{1-x} \mathrm{Sb}_{y} \mathrm{As}_{1-y}$ рассчитывались в квадратичном приближении с использованием параметров бинарных соединений InSb, InAs, $\mathrm{AlSb}$ и AlAs [9]. Распределение упругих деформаций в объеме гетероструктуры рассчитывалось в приближении сплошной среды [17] методом минимизации упругой энергии. Положения уровней размерного квантования носителей заряда рассчитывались в однозонном при- ближении. Расчеты проведены для различных значений $x_{\max }=y_{\max }$ и $x_{\min }=y_{\min }$.

Зонные диаграммы областей А и В, рассчитанные при $x_{\max }=y_{\max }=0.2$ и $x_{\min }=y_{\min }=0.15$, обеспечивающих наилучшее соответствие между энергиями оптических переходов в спектрах ФЛ и разностью вычисленных значений энергий размерного квантования электронов и дырок, представлены на рис. 6. Как видно из рисунка, области КЯ А, с низким содержанием In и

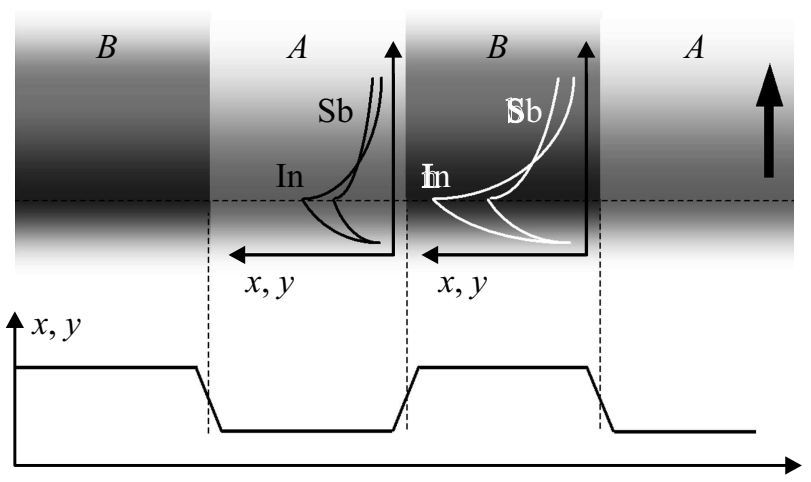

Рис. 5. Схематическое изображение поперечного сечения модельной $\operatorname{In}_{x} \mathrm{Al}_{1-x} \mathrm{Sb}_{y} \mathrm{As}_{1-y} / \mathrm{AlAs-гетероструктуры} \mathrm{в} \mathrm{об-}$ ласти КЯ. Жирная стрелка указывает направление роста структуры. Градация серого схематично отражает состав $\mathrm{In}_{x} \mathrm{Al}_{1-x} \mathrm{Sb}_{y} \mathrm{As}_{1-y}$, области с высоким содержанием In и $\mathrm{Sb}$ отмечены на рисунке как В, с низким - как А. Распределения содержания In $(x)$ и $\mathrm{Sb}(y)$ в плоскости КЯ и в направлении роста гетероструктуры приведены на нижней вставке и на центральных графиках соответственно.

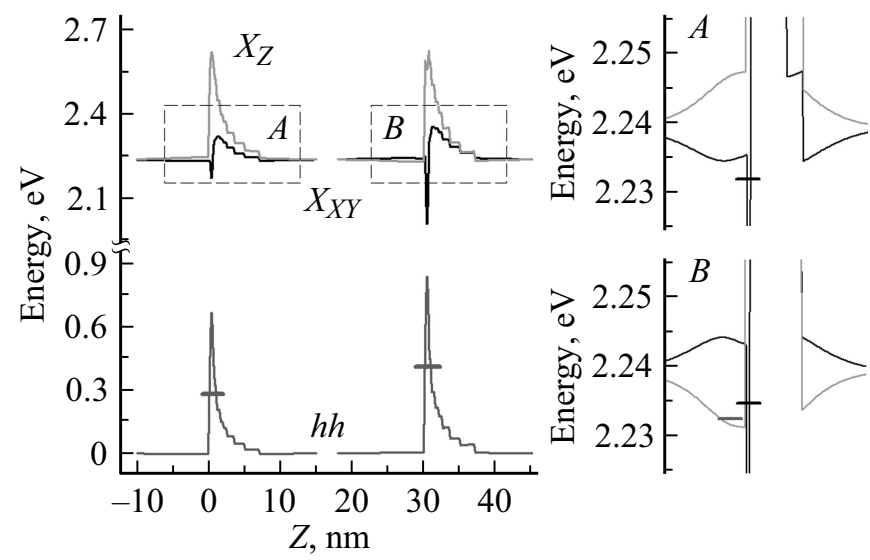

Рис. 6. Зонные диаграммы, рассчитанные для значений составов твердого раствора, дающих наилучшее соответствие между энергиями оптических переходов в спектрах ФЛ и разностью вычисленных значений энергий размерного квантования электронов и дырок. Области с высоким содержанием In и $\mathrm{Sb}$ отмечены на рисунке как В, с низким - как А. Детальная структура энергетического спектра зоны проводимости в этих областях вынесена на вставки. Края зон в $X_{Z^{-}}$и $X_{X Y}$-долинах зоны проводимости обозначены серым и черным цветом соответственно. Уровни размерного квантования электронов и дырок обозначены горизонтальными штрихами. 
$\mathrm{Sb}$, характеризуются энергетическим спектром первого рода с основным электронным состоянием, принадлежащим $X_{X Y}$-долине зоны проводимости $\operatorname{In}_{x} \mathrm{Al}_{1-x} \mathrm{Sb}_{y} \mathrm{As}_{1-y}$. В областях КЯ В, с высоким содержанием In и $\mathrm{Sb}$, энергетический спектр неоднозначен. При небольших изменениях ширины и(или) состава твердого раствора основным электронным состоянием гетероструктуры может быть либо $X_{X Y}$-состояние в зоне проводимости твердого раствора, либо $X_{Z}$-состояние в зоне проводимости AlAs-матрицы. Наблюдаемое нами в эксперименте сосуществование подсистем с энергетическим спектром первого и второго родов свидетельствует о том, что в изучаемой гетероструктуре в областях с высоким содержанием In и $\mathrm{Sb}$ основное электронное состояние принадлежит $X_{Z}$-долине зоны проводимости AlAs. Расчеты подтверждают непрямозонный характер рекомбинации носителей заряда в гетероструктуре.

Законы смещения положения максимума полос I и II от плотности возбуждения отличаются не только наличием/отсутствием слагаемого $b P_{\mathrm{ex}}^{1 / 3}$, но и величиной коэффициента $U$, описывающего сдвиг ФЛ, вызванный заполнением хвостов плотности состояний. Коэффициент $U$ для подсистемы с энергетическим спектром второго рода в $\sim 2$ раза превосходит его значение для подсистемы со спектром первого рода. Параметр $U$, как показано в работе [12], является суммой характерных энергий локализации электронов и дырок в хвостах плотности состояний Урбаха $U=U_{e}+U_{h}$. Расчеты показывают, что для областей КЯ с энергетическим спектром первого рода уровень размерного квантования электронов лежит почти на краю зоны проводимости AlAs [13]. Энергетический спектр электронов в этих областях описывается приближением „мелкой квантовой ямы“, когда положение уровня размерного квантования почти не зависит от ширины и глубины КЯ [18], поэтому их флуктуации формируют „мелкий“ хвост плотности состояний. В то же время в областях КЯ с энергетическим спектром второго рода необходимо учитывать, что флуктуации состава $\operatorname{In}_{x} \mathrm{Al}_{1-x} \mathrm{Sb}_{y} \mathrm{As}_{1-y}$ твердого раствора сопровождаются перераспределением упругих деформаций, изменяющих положение края $X_{Z}$-долины зоны проводимости в AlAs и соответственно положение уровней размерного квантования локализованных там электронов, из которых формируются хвосты плотности состояний.

\section{5. Заключение}

Изучалась кристаллическая структура и энергетический спектр гетероструктур, формирующихся при эпитаксиальном росте одного монослоя InSb в матрице AlAs. Установлено, что твердый раствор $\mathrm{In}_{x} \mathrm{Al}_{1-x} \mathrm{Sb}_{y} \mathrm{As}_{1-y}$, образующийся в процессе перемешивания материалов, при формировании КЯ в процессе эпитаксиального роста распадается на две пространственно разделенные фазы разного состава с энерге- тическим спектром первого и второго рода. Показано, что состояния электронов в областях с энергетическим строением первого и второго рода лежат в $X_{X Y^{-}}$и $X_{Z}$-долинах зоны проводимости твердого раствора и AlAs-матрицы соответственно.

Работа выполнена при финансовой поддержке правительства Российской Федерации, постановление № 211, контракт № 02.А03.21.0006; РФФИ (проекты № 1632-60015, 16-02-00242); микроскопические исследования проведены при поддержке РНФ (грант № 14-22-00143) на оборудовании ЦКП „Наноструктуры“, ФАНО РФ проект № AAАA-A17-11704210141-5.

\section{Список литературы}

[1] Z.M. Wang. Self Assembled Quantum Dots (Springer, N.Y., 2008).

[2] T.S. Shamirzaev, J. Debus, D.S. Abramkin, D. Dunker, D.R. Yakovlev, D.V. Dmitriev, A.K. Gutakovskii, L.S. Braginsky, K.S. Zhuravlev, M. Bayer. Phys. Rev. B, 84, 155318 (2011).

[3] A.V. Khaetskii, Yu.V. Nazarov. Phys. Rev. B, 64, 125316 (2001).

[4] D. Dunker, T.S. Shamirzaev, J. Debus, D.R. Yakovlev, K.S. Zhuravlev, M. Bayer. Appl. Phys. Lett., 101, 142108 (2012).

[5] T.S. Shamirzaev, J. Rautert, D.R. Yakovlev, J. Debus, A.Yu. Gornov, M.M. Glazov, E.L. Ivchenko, M. Bayer. Phys. Rev. B, 96, 035302 (2017).

[6] Т.С. Шамирзаев. ФТП, 45, 97 (2011).

[7] M.-E. Pistol, C.E. Pryor. Phys. Rev. B, 80, 035316 (2009).

[8] Д.С. Абрамкин, А.К. Бакаров, М.А. Путято, Е.А. Емельянов, Д.А. Колотовкина, А.К. Гутаковский, Т.С. Шамирзаев. ФТП, 51, 1282 (2017).

[9] I. Vurgaftman, J.R. Meyer, L.R. Ram-Mohan. J. Appl. Phys., 89, 5815 (2001).

[10] A. Boyne, S.A. Dregia, Y. Wang. Appl. Phys. Lett., 99, 063111 (2011).

[11] Н.А. Берт, Л.С. Вавилова, И.П. Ипатова, В.А. Капитонов, А.В. Мурашова, Н.А. Пихтин, А.А. Ситникова, И.С. Тарасов, В.А. Щукин. ФТП, 33, 544 (1999).

[12] D.S. Abramkin, A.K. Gutakovskii, T.S. Shamirzaev. J. Appl. Phys., 123 (11), 115701 (2018).

[13] Д.С. Абрамкин, К.М. Румынин, А.К. Бакаров, Д.А. Колотовкина, А.К. Гутаковский, Т.С. Шамирзаев. Письма ЖЭТФ, 103, 785 (2016).

[14] Д.С. Абрамкин, А.К. Бакаров, Д.А. Колотовкина, А.К. Гутаковский, Т.С. Шамирзаев. Изв. РАН, 81, 1054 (2017).

[15] K. Muraki, S. Fukatsu, Y. Shiraki, R. Ito. Appl. Phys. Lett., 61, 557 (1992).

[16] P. Offermans, P.M. Koenraad, R. Notzel, J.H. Wolter, K. Pierz. Appl. Phys. Lett., 87, 111903 (2005).

[17] C.G. Van de Walle. Phys. Rev. B, 39, 1871 (1989).

[18] Л.Д. Ландау, Е.М. Лифшиц. Теоретическая физика. Квантовая механика (нерелятивистская теория) (М., Наука. Гл. ред. физ.-мат. лит., 1989).

Редактор Г.А. Оганесян 


\section{Spinodal decomposition in InSb/AIAs heterostructures}

D.S. Abramkin ${ }^{1,2}$, A.K. Bakarov ${ }^{1}$, A.K. Gutakovskii ${ }^{1,2}$, T.S. Shamirzaev ${ }^{1,2,3}$

${ }^{1}$ Rzhanov Institute of Semiconductor Physics, Siberian Branch Russian Academy of Sciences, 630090 Novosibirsk, Russia

${ }^{2}$ Novosibirsk State University, 630090 Novosibirsk, Russia

${ }^{3}$ Ural Federal University, 620002 Yekaterinburg, Russia

Abstract Atomic structure and energy spectrum of heterostructures formed in InSb/AlAs heterosystem have been studied. The quantum well $(\mathrm{QW})$ in the heterostructure are formed from the $\operatorname{In}_{x} \mathrm{Al}_{1-x} \mathrm{Sb}_{y} \mathrm{As}_{1-y}$ alloy that dissociates in two phases with different composition. The typical size of separate alloy phase area in the structure plane is about $5-7 \mathrm{~nm}$. Spinodal decomposition of the $\mathrm{In}_{x} \mathrm{Al}_{1-x} \mathrm{Sb}_{y} \mathrm{As}_{1-y}$ alloy results in coexistent of two indirectband-gap areas inside QW with energy spectrum of type-I and type-II band alignment. 